\title{
Transcribed ultraconserved region Uc.63+ promotes resistance to cisplatin through regulation of androgen receptor signaling in bladder cancer
}

\author{
YOHEI SEKINO $^{1,2}$, NAOYA SAKAMOTO ${ }^{1}$, AKIRA ISHIKAWA ${ }^{1}$, RIRINO HONMA ${ }^{1}$, \\ YOSHINORI SHIGEMATSU ${ }^{1,2}$, TETSUTARO HAYASHI ${ }^{2}$, KAZUHIRO SENTANI $^{1}$, \\ NAOHIDE OUE $^{1}$, JUN TEISHIMA ${ }^{2}$, AKIO MATSUBARA ${ }^{2}$ and WATARU YASUI ${ }^{1}$ \\ Departments of ${ }^{1}$ Molecular Pathology, and ${ }^{2}$ Urology, Hiroshima University \\ Graduate School of Biomedical and Health Sciences, Hiroshima 734-8551, Japan
}

Received October 12,2018; Accepted February 26, 2019

DOI: $10.3892 /$ or.2019.7039

\begin{abstract}
Cisplatin (CDDP)-based combination chemotherapy is the standard for muscle-invasive bladder cancer (MIBC). However, nearly all patients undergoing CDDP chemotherapy become refractory due to the development of CDDP resistance. Therefore, clarification of the mechanisms of CDDP resistance is urgently needed. The transcribed ultraconserved regions (T-UCRs) are a novel class of non-coding RNAs that are highly conserved across species and are associated with carcinogenesis and cancer progression. In addition, emerging evidence has shown the involvement of androgen receptor (AR) signals in urothelial carcinoma (UC) progression. The aim of the present study was to investigate the expression of transcribed ultraconserved region Uc.63+, and to analyze the effects of Uc.63+ on AR expression and CDDP resistance in UC. Quantitative reverse transcription-polymerase chain reaction (qRT-PCR) revealed that the expression of Uc.63+ was higher in UC tissues than that in non-neoplastic bladder tissues and 15 types of normal tissue. An MTT assay revealed that Uc.63+ was involved in cell proliferation. Western blotting demonstrated that the expression of AR was disrupted by the overexpression or knockdown of Uc.63+ in AR-positive UMUC3 cells. Furthermore, knockdown of Uc.63+ increased sensitivity to
\end{abstract}

Correspondence to: Dr Wataru Yasui, Department of Molecular Pathology, Hiroshima University Graduate School of Biomedical and Health Sciences, 1-2-3 Kasumi, Minami-ku, Hiroshima 734-8551, Japan

E-mail: wyasui@hiroshima-u.ac.jp

Abbreviations: AR, androgen receptor; UC, urothelial carcinoma; CDDP, cisplatin; lncRNAs, long non-coding RNAs; MTT, 3-(4,5-dimethylthiazol-2-yl)-2,5-diphenyltetrazolium bromide; siRNA, small interfering RNA; T-UCR, transcribed-ultraconserved region; UMUC3-CR, CDDP-resistant UMUC3 cells

Key words: Uc.63+, bladder cancer, cell proliferation, androgen receptor, cisplatin
CDDP in UMUC3 cells. Conversely, overexpression of Uc.63+ had no effect on CDDP sensitivity in AR-negative RT112 cells. Additionally, we observed that the expression of Uc.63+ was increased in CDDP-resistant UMUC3 cells (UMUC3-CR) in comparison with that in parental UMUC3 cells. Knockdown of Uc.63+ re-sensitized the UMUC3-CR cells to CDDP. These results indicated that Uc.63+ may be a promising therapeutic target to overcome CDDP resistance in UC.

\section{Introduction}

Approximately $25 \%$ of newly diagnosed urothelial carcinoma (UC) presents as biologically aggressive muscle-invasive disease (1). Clinical studies have shown a survival benefit of neoadjuvant or adjuvant cisplatin (CDDP)-based chemotherapy for patients with muscle-invasive UC before or after radical cystectomy (2). CDDP-based chemotherapy has generally produced a complete or partial response in $\sim 50-70 \%$ of patients with UC (3). However, tumors treated with CDDP ultimately acquire CDDP resistance. Although considerable efforts have been devoted to solving CDDP resistance over the past three decades $(4,5)$, the mechanisms of CDDP resistance have yet to be fully elucidated. Therefore, clarifying new molecular mechanisms underlying CDDP resistance hold great importance in improving chemotherapy outcomes for muscle-invasive UC.

Long non-coding RNAs (IncRNAs), which are $>200 \mathrm{bp}$, are a subtype of non-coding RNAs. Although they lack protein-coding capacity, IncRNAs play an essential role in the development and progression of UC (6-8). The transcribed ultraconserved regions (T-UCRs) are a relatively new class of lncRNAs, which are highly conserved among vertebrates (9). Considering that T-UCRs are conserved across species, they allegedly play critical roles in human development and disease, including cancer. T-UCRs are differentially expressed and act in an oncogenic or tumor-suppressive role according to the context of the cancer $(10,11)$. Based on this evidence, T-UCRs may provide useful diagnostic markers for some specific types of cancer and could represent potential therapeutic targets for cancer treatment. Uc.63+ (278 bp) is 
located on the chromosome $2 \mathrm{p} 15$, in the third intron of the XPOI gene (9). A recent study revealed that Uc.63+ was induced in a hypoxia-dependent manner (12). Furthermore, the expression of Uc.63+ was revealed to be upregulated in breast cancer and was correlated with a poor prognosis (13). In addition, we previously revealed that Uc.63+ promoted docetaxel resistance through regulation of androgen receptor (AR) in prostate cancer (14). These findings indicated that Uc.63+ may contribute to progression in several types of cancers. However, there is no evidence of the role of Uc.63+ in UC. In the present study, we evaluated the expression and functional role of Uc.63+ in UC and we analyzed the effect of Uc.63+ on the expression of AR and CDDP resistance.

\section{Materials and methods}

Cell lines. Prostate cancer cell line, LNCaP, was purchased from the American Type Culture Collection (ATCC; Manassas, VA, USA). The three human UC cell lines (RT112, T24 and UMUC3) were provided by the Vancouver Prostate Centre (Vancouver, BC, Canada). Cell lines were authenticated by DNA fingerprinting using Amp FISTR Amplification or Amp FISTR Profiler PCR Amplification protocols (Life Technologies; Thermo Fisher Scientific, Inc., Waltham, MA, USA). RT112 and T24 cell lines were maintained in RPMI-1640 medium (Nissui Pharmaceutical Co. Ltd., Tokyo, Japan) containing $10 \%$ fetal bovine serum (FBS; BioWhittaker, Walkersville, MD, USA), $2 \mathrm{mM}$ L-glutamine, $50 \mathrm{U} / \mathrm{ml}$ penicillin, and $50 \mathrm{~g} / \mathrm{ml}$ streptomycin in a humidified atmosphere of $5 \% \mathrm{CO}_{2}$ and $95 \%$ air at $37^{\circ} \mathrm{C}$. LNCaP cells were withdrawn from hormone effects in the medium by culture with medium containing charcoal/dextran-stripped FBS for 2 days before treatment. Dehydrotestosteron (DHT) $(10 \mathrm{nM})$ or vehicle control $(0.5 \%$ ethanol) was added to the cells. CDDP-resistant UMUC3 cells were also provided by the Vancouver Prostate Centre. The method used to establish CDDP-resistant UMUC3 cells was previously described (15).

Tissue samples. We used 8 non-neoplastic bladder tissues and $16 \mathrm{UC}$ tissue samples for quantitative reverse transcription-polymerase chain reaction (qRT-PCR) (Table I). The samples were collected from patients at Hiroshima University Hospital (Hiroshima, Japan) from April 2010 to October 2018. The Institutional Review Board of Hiroshima University Hospital approved the present study (IRB\# E912). Appropriate written informed consent was obtained from each patient. The present study was conducted in accordance with the Ethical Guidance for Human Genome/Gene Research of the Japanese Government.

Quantitative RT-PCR analysis. Extraction of total RNA, synthesis of cDNA and qRT-PCR were performed as previously described (14). ACTB-specific PCR products, which were amplified from the same RNA samples, served as internal controls. The primer sequences and IDs are summarized in Table II.

RNA interference and expression vector. Silencer ${ }^{\circledR}$ Select (Ambion, Austin, TX, USA) against Uc.63+ was used for
Table I. Clinicopathological characteristics of $16 \mathrm{BCa}$ tissues.

Number of cases

16

Sex

Male

Median age (years)

$74(63-92)$

Race

Asian

Histology

Urothelial carcinoma

Tumor grade

G1

3

G2

4

G3

9

Pathological T stage

pT1

pT2

4

pT3

5

Pathological N stage

0

1

1

Metastasis at time of diagnosis

Absence

16

Presence

0

$\mathrm{BCa}$, bladder cancer.

RNA interference as previously described (14). Transfection was performed using Lipofectamine RNAiMAX (Invitrogen; Thermo Fisher Scientific, Inc.) according to the manufacturer's instructions. Cells were used $48 \mathrm{~h}$ after transfection in each of the experiments and assays.

For constitutive expression of Uc.63+, cDNA was PCR amplified and subcloned into pcDNA 3.1 (Invitrogen; Thermo Fisher Scientific, Inc.) as previously described (14). The pcDNA-Uc.63+ expression vector was transfected into RT112 and UMUC3 cells with FuGENE6 (Roche Diagnostics, Basel, Switzerland) according to the manufacturer's instructions.

For constitutive expression of human AR, cDNA was PCR amplified from normal prostate tissues and subcloned into pDON-5 Neo (Takara Biotechnology Co., Ltd., Dalian, China) using a retrovirus vector with psPAX2 envelope and pMD2.G packaging plasmids, according to the manufacturer's instructions.

Cell proliferation assay. To examine cell proliferation, an MTT assay was performed as previously described (16). Cell proliferation was monitored after 1,2 and 4 days.

Cell death ELISA. Cells were seeded in 12-well plates ( $1 \times 10^{5}$ cells). Mononucleosomes and oligonucleosomes in the cytoplasmic fraction were assessed by a Cell Death Detection ELISA kit (Roche Diagnostics) according to the manufacturer's instructions. Absorbance was determined at $405 \mathrm{~nm}$. 
Table II. Primer sequences for qRT-PCR.

Forward primer

Reverse primer

\begin{tabular}{ll} 
Uc.63+ & TTGCATAAAAGCCAAATGTCA \\
AR & GACGCTTCTACCAGCTCACC \\
ACTB & TCACCGAGCGCGGCT \\
\hline
\end{tabular}

CTGTTTGCTTGCCTGGTAAA

GAAAGGATCTTGGGCACTTG

ACTB

TCACCGAGCGCGGCT

TAATGTCACGCACGATTTCCC

$\mathrm{AR}$, androgen receptor; ACTB, $\beta$-actin.

Western blot analysis. For western blot analysis, cells were lysed as previously described (17). Proteins in UC cell lines were extracted using RIPA buffer and subjected to concentration measurements using the BCA kit. The lysates $(40 \mu \mathrm{g})$ were solubilized in Laemmli sample buffer by boiling and then subjected to $10 \%$ SDS-polyacrylamide gel electrophoresis followed by electrotransfer onto a nitrocellulose membrane. Cleaved PARP (c-PARP) (cat. no. 9542; Cell Signaling Technology, Inc., Danvers, MA, USA) and AR antibody (cat. no. MA5-13423; Thermo Fisher Scientific, Inc.) were used as the primary antibodies with a 1:1,000 dilution. $\beta$-actin (Sigma-Aldrich; Merck KGaA, Darmstadt, Germany) was detected as a loading control. Peroxidase-conjugated anti-mouse IgG (cat. no. 330; MBL, Nagoya, Japan) or anti-rabbit IgG (cat. no. 458; MBL) were used as the secondary antibodies with a 1:500 dilution. Immunocomplexes were visualized with an ECL Western Blot Detection system (Amersham Biosciences; GE Healthcare, Chicago, IL, USA).

Drug treatment. CDDP (Nippon Kayaku Co., Ltd., Tokyo, Japan) was obtained and handled according to the manufacturer's recommendations. Cell lines treated with vehicle ( $0.5 \%$ ethanol) or escalating doses of CDDP were assessed for cell viability. An MTT assay was performed at $48 \mathrm{~h}$ after CDDP chemotherapy (15). Drug sensitivity curves and $\mathrm{IC}_{50}$ values were calculated using GraphPad Prism 4.0 software (GraphPad Software Inc., San Diego, CA, USA).

Statistical analysis. All experiments were repeated at least three times with each sample in triplicate. The results are expressed as the mean \pm standard deviation (SD) of triplicate measurements. Sample sizes for relevant experiments were determined by power analysis. Statistical differences were evaluated using the Mann-Whitney U test. A P-value of $<0.05$ was considered to indicate a statistically significant result. One-way analysis of variance (ANOVA) followed by Tukey's test was used for the comparison of multiple groups. Spearman's correlation coefficient was used to examine the degree of relations between two groups. Statistical analyses were conducted primarily using GraphPad Prism software (version 4.0; GraphPad Software Inc., La Jolla, CA, USA).

\section{Results}

Expression of Uc.63+ is upregulated in UC tissues and UC cell lines. We analyzed the expression of Uc.63+ in 16 UC tissues and 8 non-neoplastic urinary bladder tissues using qRT-PCR. The expression of Uc.63+ was upregulated in $69 \%(11 / 16)$ of UC tissues compared with that in non-neoplastic urinary bladder tissues ( $\mathrm{P}=0.001$; Fig. 1A). Despite the small sample sets, the expression of Uc.63+ was significantly higher in muscle invasive UC than that in non-muscle invasive $\mathrm{UC}(\mathrm{P}=0.047)$ (Fig. 1B). As revealed in Fig. 1C, the expression of Uc.63+ was higher in UC tissues and UC cell lines than that in the 15 types of normal tissue samples including a normal urinary bladder tissue.

Uc.63+ acts as an oncogene and is associated with cell proliferation and apoptosis. To clarify the biological roles of Uc.63+ in UC, we investigated the effects on the knockdown of Uc.63+ using small interfering RNA (siRNA) that was previously designed to specifically target Uc.63+ (14). We confirmed a significantly lower expression of Uc.63+ with two different siRNAs than with the negative control in UMUC3 cells (Fig. 2A). Next, we performed a 3-(4,5-dimethylthiazol-2-yl)-2,5-diphenyltetrazolium bromide (MTT) assay and observed that the downregulation of Uc.63+ significantly suppressed cell proliferation of UMUC3 (Fig. 2B). A recent study revealed that Uc.63+ induced apoptosis in breast cancer (13). Therefore, we analyzed the effect of knockdown of Uc.63+ on apoptosis. Cell d eath ELISA revealed that knockdown of Uc.63+ significantly induced apoptosis compared to the negative control (Fig. 2C). Western blotting revealed that knockdown of Uc.63+ enhanced the expression of cleaved PARP which was used as the activation status of apoptosis (Fig. 2D). To further verify whether Uc.63+ plays an important role in cell proliferation and apoptosis, we transfected the Uc.63+ expression vector into RT112, which had low expression of Uc.63+. As anticipated, the MTT assay revealed that the overexpression of Uc.63+ significantly increased cell proliferation (Fig. 2E and F). Cell death ELISA revealed that the overexpression of Uc.63+ significantly suppressed apoptosis compared to the empty vector (Fig. 2G). These results indicated that Uc.63+ was involved in cell proliferation and apoptosis in UC.

Uc.63+ modulates the expression of AR. Several studies have reported that AR is involved in the development and progression of UC $(18,19)$. Previously, we revealed that overexpression of Uc.63+ enhanced the expression of AR (14). Given these findings, we analyzed the association between Uc.63+ and AR in UC. We investigated the expression of AR in $16 \mathrm{UC}$ tissues by qRT-PCR and found that the expression of AR was significantly correlated with the expression of Uc.63+ $(\mathrm{P}<0.001$, $\mathrm{R}=0.74$ ) (Fig. 3A). Western blotting revealed that the expression of AR was detected in UMUC3 cells, which was consistent with 

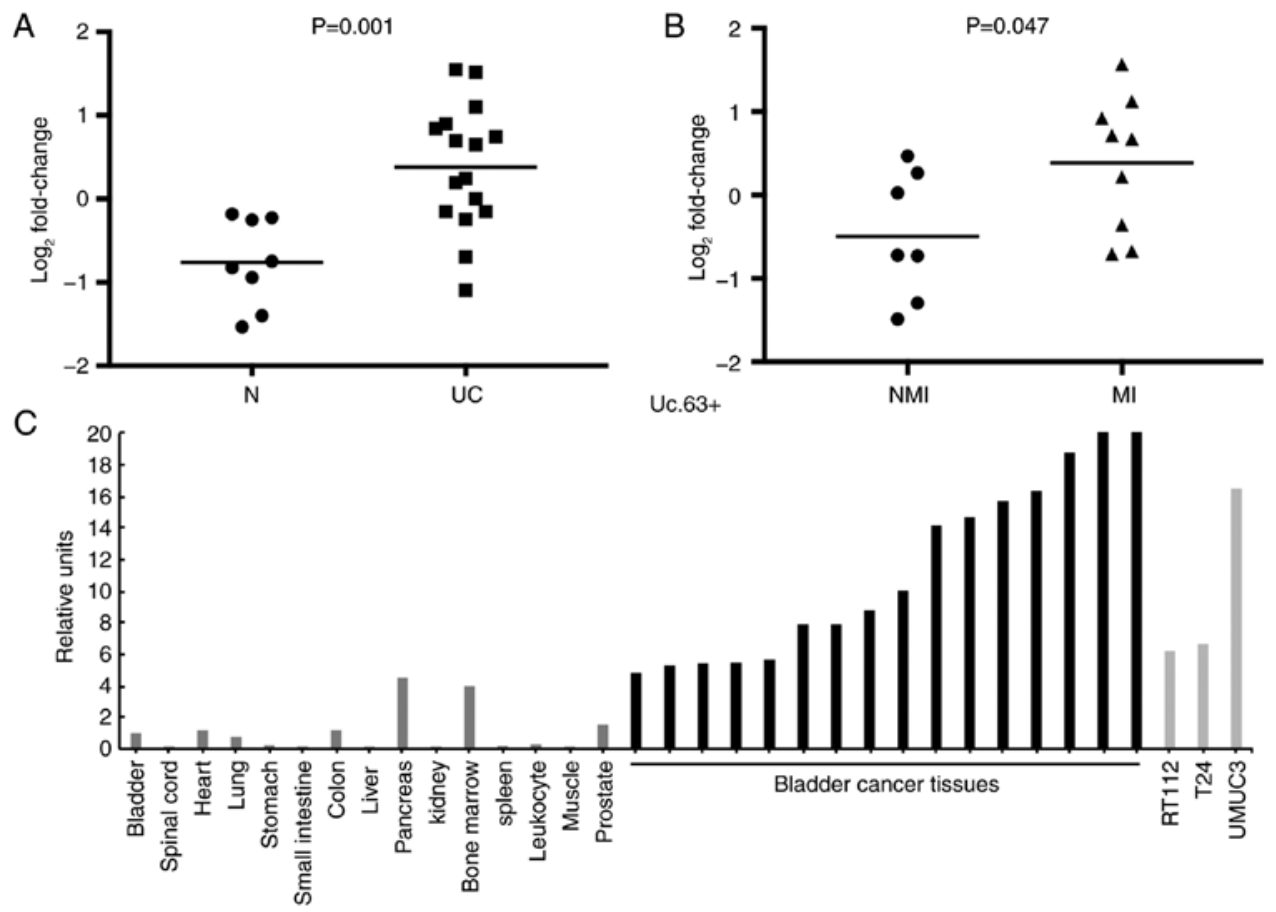

Figure 1. The expression of Uc.63+ in UC. (A) The results of qRT-PCR analysis for the expression of Uc.63+ in 16 UC tissues and normal bladder tissues (N). Statistical differences were evaluated with the Mann-Whitney U test. (B) Scatter plot diagrams showing the expression of Uc.63+ in 7 non-muscle invasive UC and 9 muscle invasive UC. Statistical differences were evaluated with the Mann-Whitney U test. (C) qRT-PCR analysis of the expression of Uc.63+ in 15 types of normal tissue, 16 UC samples, and 3 human UC cell lines (RT112, T24 and UMUC3). UC, urothelial carcinoma; NMI, non-muscle invasive UC tissues; MI, muscle invasive UC tissues; qRT-PCR, quantitative reverse transcription-polymerase chain reaction.
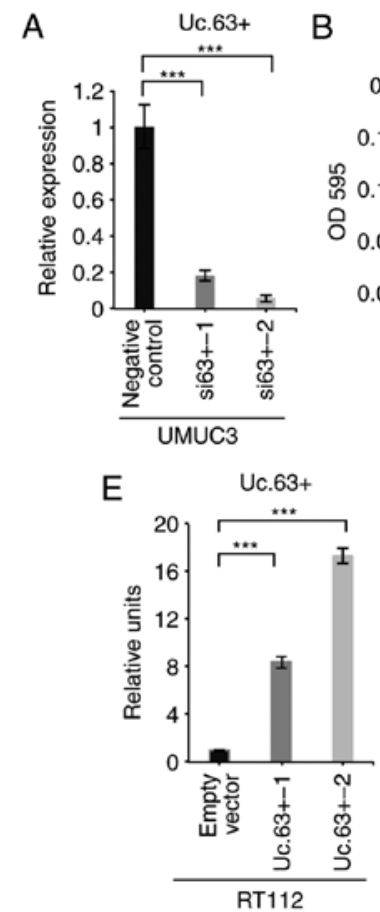

B

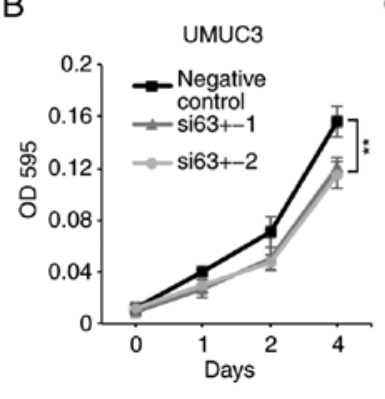

$\mathrm{F}$

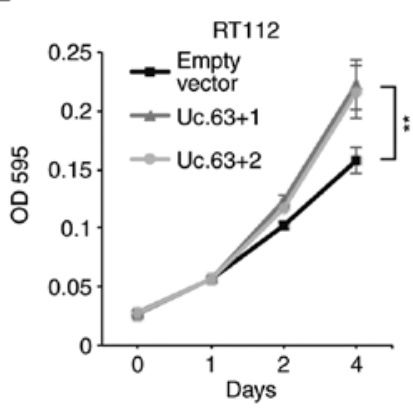

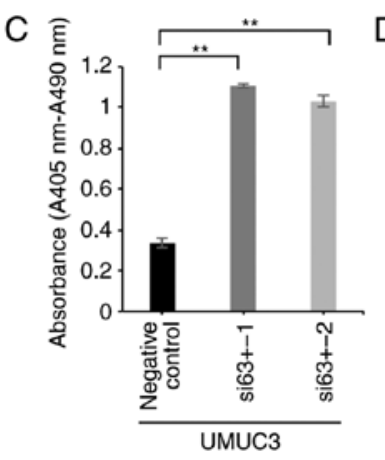
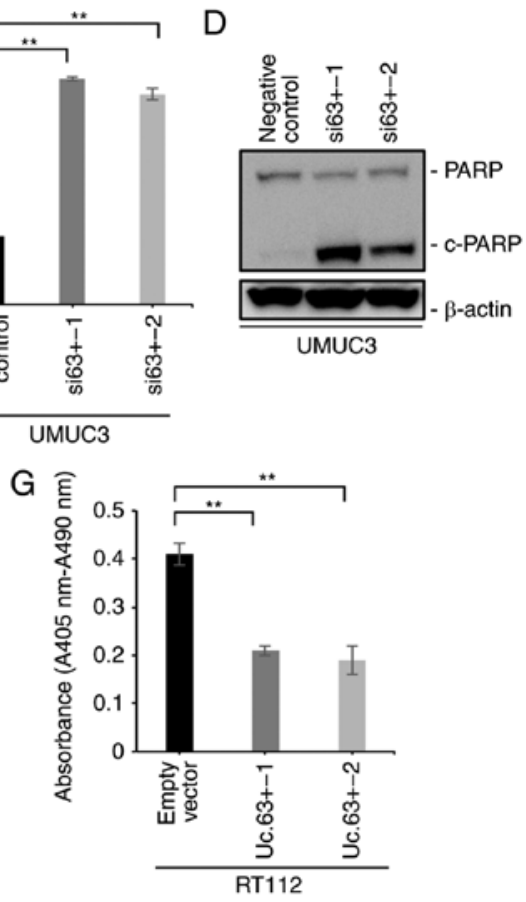

Figure 2. Uc.63+ promotes cell proliferation in UC cells. (A) qRT-PCR results for the expression of Uc.63+ in UMUC3 cells transfected with negative control or two different siRNAs. The results are expressed as the mean and standard deviation (SD) of triplicate measurements. ${ }^{* * *} \mathrm{P}<0.001$. (B) Cell proliferation assay in UMUC3 cells transfected with the negative control or two different siRNAs. Cell proliferation was assessed by MTT assays at 1,2 and 4 days after seeding on 96-well plates. Bars and error bars are the mean and SD, respectively, of 3 independent experiments ${ }^{* *} \mathrm{P}<0.01$. (C) The cell death ELISA in UMUC3 cells transfected with negative control or two different siRNAs. The results are expressed as the mean and standard deviation (SD) of triplicate measurements. ${ }^{* *} \mathrm{P}<0.01$. (D) Western blotting of PARP and c-PARP in UMUC3 cells. $\beta$-actin was used as a loading control. (E) The results of qRT-PCR for the expression of Uc.63+ in RT112 cells transfected with empty vector or Uc.63+ expression vector. The results are expressed as the mean and standard deviation (SD) of triplicate measurements. ${ }^{* * *} \mathrm{P}<0.001$. (F) Cell proliferation assay in RT112 cells transfected with empty vector or Uc.63+ expression vector. Cell proliferation was assessed by MTT assays at 1,2 and 4 days after seeding on 96-well plates. Bars and error bars are the mean and SD, respectively, of 3 independent experiments. ${ }^{* *} \mathrm{P}<0.01$. (G) The cell death ELISA in RT112 cells transfected with empty vector or Uc.63+ expression vector. The results are expressed as the mean and standard deviation (SD) of triplicate measurements. ${ }^{* *} \mathrm{P}<0.01$. UR, urothelial carcinoma; c-PARP, cleaved PARP; qRT-PCR, quantitative reverse transcription-polymerase chain reaction. 
A

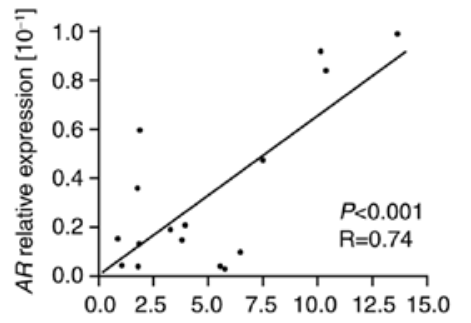

UC. $63+$ relative expression $\left[10^{-3}\right]$
B

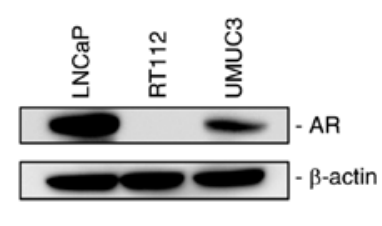

C

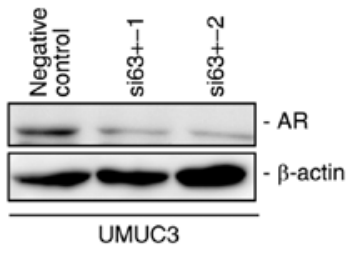

D

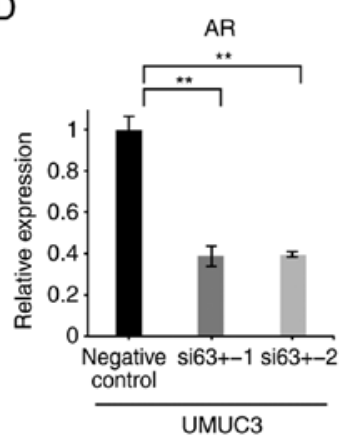

E

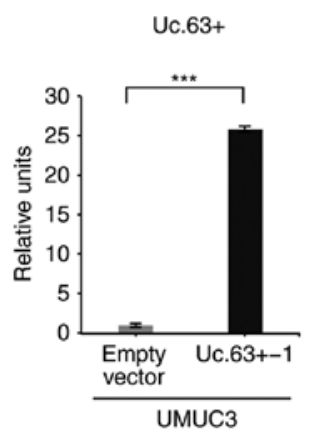

G

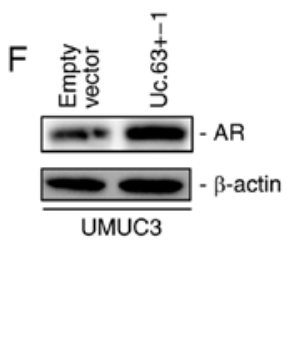

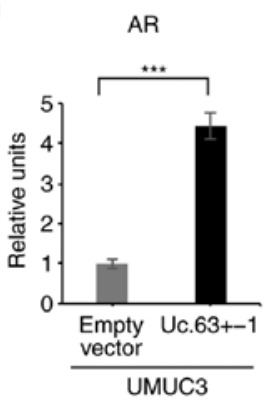

Figure 3. Uc.63+ modulates the expression of AR. (A) qRT-PCR analysis for the correlation between Uc.63+ and AR in bladder cancer tissues. Spearman correlation coefficient and P-values are indicated. (B) Western blotting of AR in LNCaP, RT112 and UMUC3 cells. $\beta$-actin was used as a loading control. (C) Western blotting of AR in UMUC3 cells transfected with negative control or two different siRNAs. $\beta$-actin was used as a loading control. (D) qRT-PCR analysis for the expression of AR in UMUC3 cells transfected with negative control or two different siRNAs. The results are expressed as the mean and standard deviation (SD) of triplicate measurements. ${ }^{* *} \mathrm{P}<0.01$. (E) qRT-PCR analysis for the expression of Uc.63+ in UMUC3 cells transfected with empty vector or Uc.63+ expression vector. The results are expressed as the mean and standard deviation (SD) of triplicate measurements. ${ }^{* * *} \mathrm{P}<0.001$. (F) Western blotting of AR in UMUC3 cells transfected with empty vector or Uc.63+ expression vector. $\beta$-actin was used as a loading control. (G) qRT-PCR analysis for the expression of AR in UMUC3 cells transfected with empty vector or Uc.63+ expression vector. The results are expressed as the mean and standard deviation (SD) of triplicate measurements. ${ }^{* * * *} \mathrm{P}<0.001$. AR, androgen receptor; siRNA, small interfering RNA; qRT-PCR, quantitative reverse transcription-polymerase chain reaction.

previous studies $(19,20)$ (Fig. 3B). To further investigate the interaction between Uc.63+ and AR, we examined the effect of Uc.63+ deregulation on the expression of AR. Knockdown of Uc.63+ suppressed the expression of AR in UMUC3 cells at the mRNA and protein levels (Fig. 3C and D). We confirmed a significantly higher expression of Uc.63+ with Uc.63+ expression vector than with the empty vector in UMUC3 cells (Fig. 3E). Overexpression of Uc.63+ induced the expression of AR in UMUC3 cells at the mRNA and protein levels in UMUC3 cell lines (Fig. 3F and G). In addition, upregulation of Uc.63+ did not affect the expression of AR in RT112 cells (data not shown). These results indicated that Uc.63+ modulated the expression of AR in UMUC3 cells.

Uc.63+ promotes CDDP resistance through AR. A recent study revealed that AR activity modulated CDDP sensitivity (21). We transfected the AR expression vector into RT112 cells, which do not express AR (Fig. 3B). The expression of AR was upregulated with AR expression vector compared to that with the empty vector in RT112 cells as determined by western blotting (Fig. 4A). We measured cell viability in RT112 cells with empty vector or AR expression vector under $0,5,10,20$ and $40 \mu \mathrm{M}$ of CDDP using an MTT assay. Overexpression of AR decreased CDDP sensitivity in RT112 cells (Fig. 4B), which was consistent with a previous study (21). To clarify the effect of Uc.63+ on CDDP resistance, we measured the cell viability in UMUC3 cells with deregulation of Uc.63+ under $0,5,10,20$ and $40 \mu \mathrm{M}$ of CDDP using an MTT assay. The $\mathrm{IC}_{50}$ value of UMUC3 cells transfected with the negative control was higher than that of UMUC3 cells transfected with siRNAs for Uc.63+ (Fig. 4C). As anticipated, overexpression of Uc.63+ promoted CDDP resistance in UMUC3 cells (Fig. 4D). Conversely, overexpression of Uc.63+ did not affect CDDP sensitivity in RT112 cells (Fig. 4E). Collectively, these results indicated that Uc.63+ may in some way be involved in the acquisition of CDDP resistance through AR regulation.

Inhibition of $U c .63+$ reverses $C D D P$ resistance. We previously established CDDP-resistant UMUC3 cells (UMUC3-CR) by long-term culture with low to increasing doses of CDDP (15) and confirmed that UMUC3-CR cells were more resistant to CDDP than were parental UMUC3 cells (data not shown). Recent evidence demonstrated that the expression of $\mathrm{AR}$ was enhanced in CDDP-resistant UC cell lines (21). Western blotting revealed that the expression of AR was upregulated in UMUC3-CR cells compared with that in parental UMUC3 cells (Fig. 5A). Furthermore, we investigated the involvement of Uc.63+ in CDDP resistance. qRT-PCR revealed that the expression of Uc.63+ was higher in UMUC3-CR cells than in parental UMUC3 cells (Fig. 5B). We measured the cell viability in UMUC3-CR cells with knockdown of Uc.63+ under 0, 5, 10, 20, 40 and $80 \mu \mathrm{M}$ of CDDP. The $\mathrm{IC}_{50}$ value of UMUC3-CR cells transfected with the negative control was higher than that of UMUC3 cells transfected with siRNAs (Fig. 5C). Collectively, these results indicated that inhibition of Uc.63+ re-sensitized the UMUC3-CR cells to CDDP. 

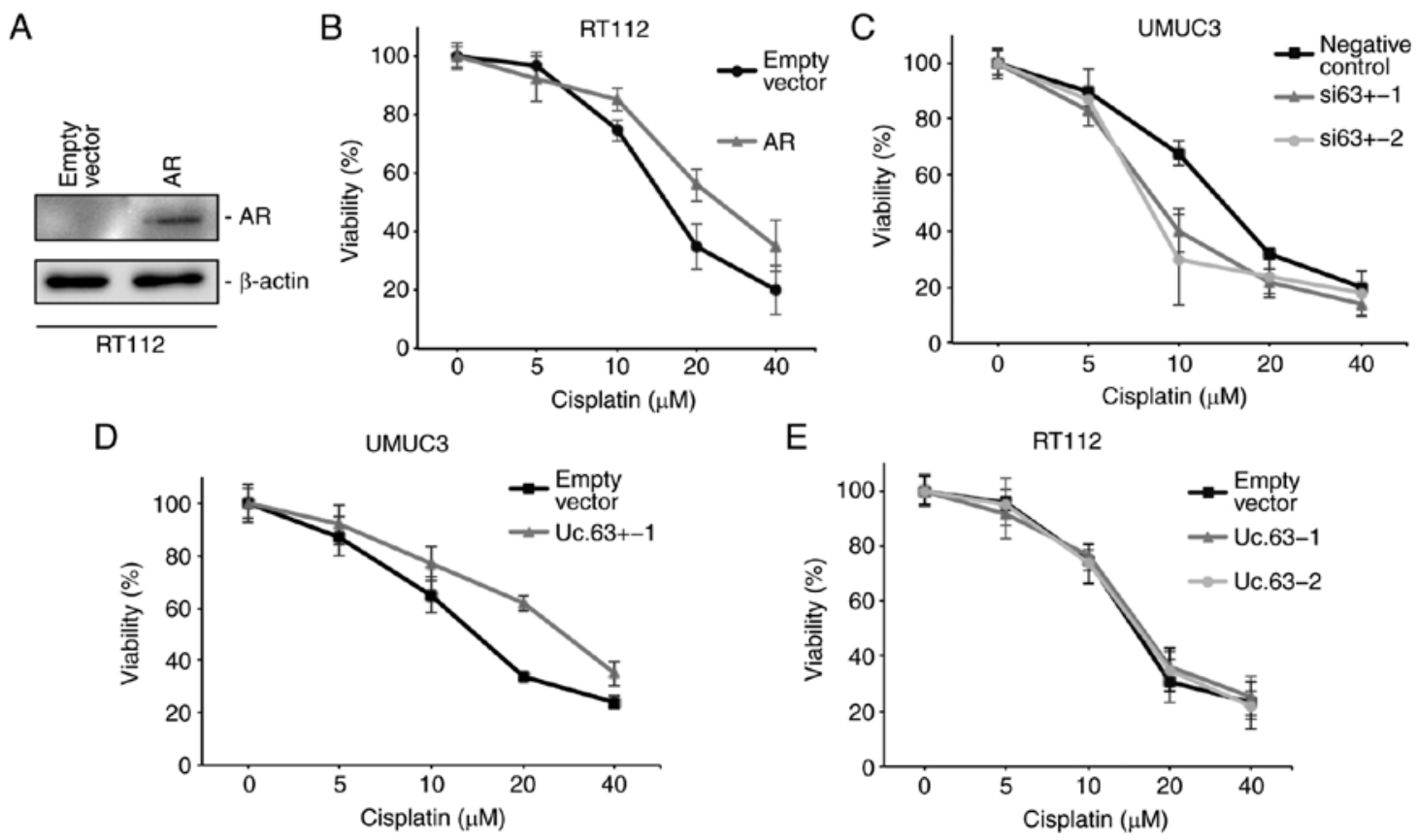

Figure 4. Effect of Uc.63+ on cisplatin sensitivity in bladder cancer cell lines. (A) Western blotting of AR in RT112 cells transfected with empty vector or AR expression vector. $\beta$-actin was used as a loading control. (B) Dose-dependent effect of cisplatin on the viability of RT112 cells transfected with empty vector or AR expression vector. The $\mathrm{IC}_{50}$ value of the empty vector, $15.3 \mu \mathrm{M}$; the $\mathrm{IC}_{50}$ value of $\mathrm{AR}, 25.9 \mu \mathrm{M}$. (C) Dose-dependent effect of cisplatin on the viability of UMUC3 cells transfected with negative control or two different siRNAs. The $\mathrm{IC}_{50}$ of the negative control, $14.1 \mu \mathrm{M}$; the $\mathrm{IC}_{50}$ value of si63+-1, $8.5 \mu \mathrm{M}$; the $\mathrm{IC}_{50}$ value of si63+-2, $7.7 \mu \mathrm{M}$. (D) Dose-dependent effect of cisplatin on the viability of UMUC3 cells transfected with empty vector or Uc.63+ expression vector. The $\mathrm{IC}_{50}$ value of the empty vector, $13.9 \mu \mathrm{M}$; the $\mathrm{IC}_{50}$ value of Uc.63+-1, $27.1 \mu \mathrm{M}$. (E) Dose-dependent effect of CDDP on the viability of RT112 cells transfected with empty vector or Uc.63+ expression vector. The $\mathrm{IC}_{50}$ value of the empty vector, $14.7 \mu \mathrm{M}$, the $\mathrm{IC}_{50}$ value of $\mathrm{Uc} .63+-1,15.6 \mu \mathrm{M}$; the $\mathrm{IC}_{50}$ value of Uc.63+-2, $15.2 \mu \mathrm{M}$. AR, androgen receptor; siRNA, small interfering RNA.

A
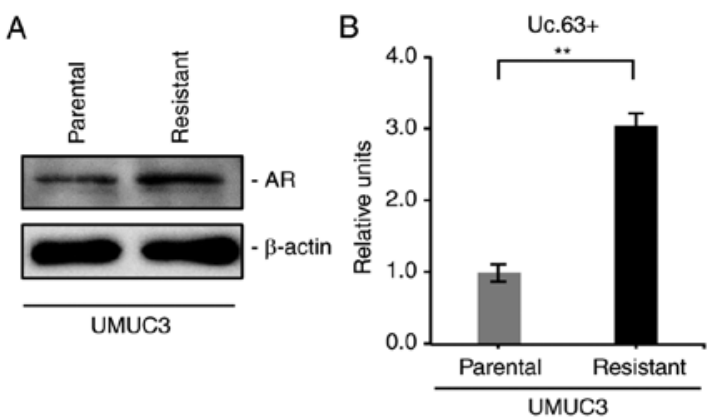

C

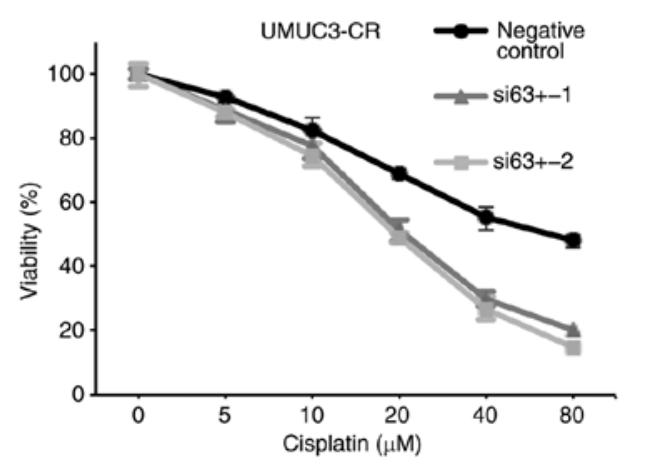

Figure 5. Downregulation of Uc.63+ re-sensitizes cisplatin-resistant UMUC3 cells to cisplatin. (A) Western blotting of AR in parental UMUC3 cells and UMUC3-CR cells. $\beta$-actin was used as a loading control. (B) qRT-PCR analysis of the expression of Uc.63+ in parental UMUC3 cells and UMUC3-CR cells. The results are expressed as the mean and standard deviation (SD) of triplicate measurements. ${ }^{* *} \mathrm{P}<0.01$. (C) Dose-dependent effect of cisplatin on the viability of UMUC3-CR cells transfected with negative control or two different siRNAs. The $\mathrm{IC}_{50}$ value of the negative control, $65.6 \mu \mathrm{M}$; the $\mathrm{IC}_{50}$ value of si63+-1, $20.7 \mu \mathrm{M}$; the $\mathrm{IC}_{50}$ value of si63+-2, $19.5 \mu \mathrm{M}$. AR, androgen receptor; UMUC3-CR, CDDP-resistant UMUC3 cells; qRT-PCR, quantitative reverse transcription-polymerase chain reaction.

\section{Discussion}

Transcribed ultraconserved regions (T-UCRs) are highly conserved among most of the vertebrates, implying that T-UCRs could play an important role in biological processes compared with other non-coding RNAs (9). Several recent studies have shown that cancer-specific T-UCRs are involved in cancer progression and tumorigenesis in some types of cancer $(11,22)$. To date, it has been reported that upregulation of Uc.8+ increased the expression of MMP9 and promoted cancer progression in urothelial carcinoma (UC) (23). However, the role of T-UCRs in UC remains unclear. The present study, which is the first, to the best of our knowledge, to investigate the expression and biological role of Uc.63+ in UC, revealed that the expression of Uc.63+ was upregulated in UC compared with 15 types of normal tissue samples including normal bladder tissue. In addition, we revealed that Uc.63+ was involved in cell proliferation and apoptosis in vitro. These results indicated that Uc.63+ was more likely to contribute to cancer progression than tumorigenesis in UC.

Some lines of evidence have recently indicated that androgen receptor (AR) signaling plays a pivotal role in carcinogenesis and cancer progression in UC $(20,24)$. Retrospective clinical studies have revealed that androgen deprivation therapy prevents the recurrence of UC in male patients $(25,26)$. One recent study revealed that lncRNA XIST interacted with miR-124 to promote cell proliferation, invasion and migration through AR in UC (27). Although the essential role of AR in the development and progression of UC has 
been reported, little evidence is available with regard to the regulation of AR in UC. In the present study, the expression of AR was significantly disrupted by the overexpression or knockdown of Uc.63+. Furthermore, qRT-PCR revealed that there was a positive association between Uc.63+ and AR in UC tissues. Previously, we revealed that Uc.63+ modulated AR expression in prostate cancer (14). Collectively, these data indicated that Uc.63+ may play an important role in the AR pathway in both UC and prostate cancer. However, the mechanism of how Uc.63+ regulates the expression of AR remains unclear. To date, there is well known evidence indicating that microRNA-T-UCR interactions contribute to tumorigenesis and cancer progression in some types of cancer $(22,28)$. There may be numerous miRNAs that are potentially regulated by Uc.63+, which may explain how Uc.63+ regulates the expression of AR. Although further studies are required to elucidate the mechanism of regulation of AR by Uc.63+, the interaction between Uc.63+ and AR may play a decisive role in cancer progression in UC.

Cisplatin (CDDP) has been widely used for the treatment of muscle-invasive UC. However, the elevated incidence of CDDP resistance is the main obstacle in clinical practice. Molecular mechanisms of CDDP resistance are believed to be caused by multiple factors including drug transport, detoxification, DNA repair and apoptosis $(4,5)$. A recent study revealed that $A R$ activation resulted in induction of $C D D P$ resistance and modulated CDDP sensitivity (21). Another study reported that the expression of AR was increased in CDDP-resistant cell lines in endometrial carcinoma (29). In the presents study, we revealed that upregulation of AR decreased CDDP sensitivity in RT112 cells, which is an AR-negative cell line, and that the disrupted expression of Uc.63+ modulated CDDP sensitivity in UMUC3 cells, which is an AR-positive cell line. Overexpression of Uc.63+ had no effect on CDDP sensitivity in RT112 cells. Collectively, these results indicated that Uc.63+ may be responsible for CDDP resistance through AR in UC. To the best of our knowledge, these findings represent the first evidence that CDDP resistance can be regulated by a specific T-UCR. In current cancer treatments, different types of chemotherapeutic agents are often combined to improve efficacy and to minimize toxicity. In the present study, inhibition of Uc.63+ re-sensitized UMUC3-CR cells to CDDP treatment. Furthermore, we observed that the expression of Uc.63+ was higher in UC tissues than in various normal tissue samples, indicating that the combination therapy of a Uc.63 inhibitor and CDDP may be a promising strategy to overcome CDDP resistance with fewer adverse effects.

There are some limitations in the present study. One of the biggest issues of the present study was that the sample size for Uc.63+ expression by qRT -PCR was relatively small, which signifies that our results may not apply to most patients with UC. Therefore, a study with a larger number of patients with UC will be necessary to further verify this current data. The other issue is that we could not show solid evidence that underpins the involvement of Uc.63+ in apoptosis. In order to confirm this novelty, validation of the effect of modulation of Uc.63+ on cell cycle distribution and morphological change in addition to cell death ELISA and analysis of the expression of c-PARP should be performed.
In summary, our results revealed that the expression of Uc.63+ was upregulated in UC tissues and UC cell lines. Uc.63+ acted as an oncogene and was associated with cell proliferation. We also revealed that Uc.63+ modulated the expression of AR and promoted CDDP resistance through AR. Furthermore, inhibition of Uc.63+ reversed CDDP resistance in vitro. The data presented here highlight the potential of Uc.63+ as a therapeutic target in patients with CDDP treatment.

\section{Acknowledgements}

We would like to thank Mr. Shinichi Norimura for his excellent technical assistance. The present study was carried out with the kind cooperation of the Research Center for Molecular Medicine of the Faculty of Medicine of Hiroshima University. We also thank the Analysis Center of Life Science of Hiroshima University for the use of their facilities.

\section{Funding}

The present study was supported by Grants-in-Aid for Scientific Research (nos. JP15H04713 and JP16K08691) and by the Challenging Exploratory Research (nos. 26670175 and JP16K15247) from the Japan Society for the Promotion of Science.

\section{Availability of data and materials}

All data generated or analyzed during the present study are included in this article.

\section{Authors' contributions}

YSe, NS, KS, NO and WY designed the study. YSh, AI, TH and AM provided the patients' clinical information. YSe, RH and JT performed the experiments and acquired the data. YSh, NS, AI, KS, NO and WY interpreted the results. YSe, TH, NS $\mathrm{KS}, \mathrm{NO}, \mathrm{JT}, \mathrm{AM}$ and WY drafted and edited the manuscript. All authors read and approved the manuscript and agree to be accountable for all aspects of the research in ensuring that the accuracy or integrity of any part of the work are appropriately investigated and resolved.

\section{Ethics approval and consent to participate}

The Institutional Review Board of Hiroshima University Hospital approved the present study (IRB\# E912). Appropriate written informed consent was obtained from each patient. The present study was conducted in accordance with the Ethical Guidance for Human Genome/Gene Research of the Japanese Government.

\section{Patient consent for publication}

Not applicable.

\section{Competing interests}

The authors declare that they have no competing interests. 


\section{References}

1. Lobo N, Mount C, Omar K, Nair R, Thurairaja R and Khan MS Landmarks in the treatment of muscle-invasive bladder cancer. Nat Rev Urol 14: 565-574, 2017.

2. Keegan KA, Zaid HB, Patel SG and Chang SS: Increasing utilization of neoadjuvant chemotherapy for muscle-invasive bladder cancer in the United States. Curr Urol Rep 15: 394, 2014.

3. von der Maase H, Sengelov L, Roberts JT, Ricci S, Dogliotti L, Oliver T, Moore MJ, Zimmermann A and Arning M: Long-term survival results of a randomized trial comparing gemcitabine plus cisplatin, with methotrexate, vinblastine, doxorubicin, plus cisplatin in patients with bladder cancer. J Clin Oncol 23: 4602-4608, 2005.

4. Amable L: Cisplatin resistance and opportunities for precision medicine. Pharmacol Res 106: 27-36, 2016.

5. Galluzzi L, Vitale I, Michels J, Brenner C, Szabadkai G, HarelBellan A, Castedo M and Kroemer G: Systems biology of cisplatin resistance: Past, present and future. Cell Death Dis 5: e1257, 2014.

6. Martens-Uzunova ES, Bottcher R, Croce CM, Jenster G, Visakorpi $\mathrm{T}$ and Calin GA: Long noncoding RNA in prostate, bladder, and kidney cancer. Eur Urol 65: 1140-1151, 2014.

7. Chandra Gupta $S$ and Nandan Tripathi Y: Potential of long non-coding RNAs in cancer patients: From biomarkers to therapeutic targets. Int J Cancer 140: 1955-1967, 2017.

8. Taheri M, Omrani MD and Ghafouri-Fard S: Long non-coding RNA expression in bladder cancer. Biophys Rev 10: 1205-1213, 2017.

9. Bejerano G, Pheasant M, Makunin I, Stephen S, Kent WJ, Mattick JS, Haussler D: Ultraconserved elements in the human genome. Science 304: 1321-1325, 2004.

10. Lujambio A, Portela A, Liz J, Melo SA, Rossi S, Spizzo R, Croce CM, Calin GA and Esteller M: CpG island hypermethylation-associated silencing of non-coding RNAs transcribed from ultraconserved regions in human cancer. Oncogene 29: 6390-6401, 2010.

11. Goto K, Ishikawa S, Honma R, Tanimoto K, Sakamoto N, Sentani K, Oue N, Teishima J, Matsubara A and Yasui W: The transcribed-ultraconserved regions in prostate and gastric cancer: DNA hypermethylation and microRNA-associated regulation. Oncogene 35: 3598-3606, 2016.

12. Ferdin J, Nishida N, Wu X, Nicoloso MS, Shah MY, Devlin C, Ling H, Shimizu M, Kumar K, Cortez MA, et al: HINCUTs in cancer: Hypoxia-induced noncoding ultraconserved transcripts. Cell Death Differ 20: 1675-1687, 2013.

13. Marini A, Lena AM, Panatta E, Ivan C, Han L, Liang H, Annicchiarico-Petruzzelli M, Di Daniele N, Calin GA, Candi E, et al: Ultraconserved long non-coding RNA uc.63 in breast cancer. Oncotarget 8: 35669-35680, 2017.

14. Sekino Y, Sakamoto N, Goto K, Honma R, Shigematsu Y, Sentani K, Oue N, Teishima J, Matsubara A and Yasui W: Transcribed ultraconserved region Uc.63+ promotes resistance to docetaxel through regulation of androgen receptor signaling in prostate cancer. Oncotarget 8: 94259-94270, 2017.

15. Hayashi T, Seiler R, Oo HZ, Jäger W, Moskalev I, Awrey S, Dejima T, Todenhöfer T, Li N, Fazli L, et al: Targeting HER2 with T-DM1, an antibody cytotoxic drug conjugate, is effective in HER 2 over expressing bladder cancer. J Urol 194: 1120-1131, 2015.
16. Oue N, Naito Y, Hayashi T, Takigahira M, Kawano-Nagatsuma A, Sentani K, Sakamoto N, Zarni Oo H, Uraoka N, Yanagihara K, et al: Signal peptidase complex 18, encoded by SEC11A, contributes to progression via TGF-alpha secretion in gastric cancer. Oncogene 33: 3918-3926, 2014.

17. Sekino Y, Oue N, Shigematsu Y, Ishikawa A, Sakamoto N, Sentani K, Teishima J, Matsubara A and Yasui W: KIFC1 induces resistance to docetaxel and is associated with survival of patients with prostate cancer. Urol Oncol 35: 31 e13-31 e20, 2017.

18. Miyamoto H, Zheng Y and Izumi K: Nuclear hormone receptor signals as new therapeutic targets for urothelial carcinoma. Curr Cancer Drug Targets 12: 14-22, 2012.

19. Miyamoto H, Yang Z, Chen YT, Ishiguro H, Uemura H, Kubota Y, Nagashima Y, Chang YJ, Hu YC, Tsai MY, et al: Promotion of bladder cancer development and progression by androgen receptor signals. J Natl Cancer Inst 99: 558-568, 2007.

20. Shiota M, Takeuchi A, Yokomizo A, Kashiwagi E, Tatsugami K, Kuroiwa $\mathrm{K}$ and Naito S: Androgen receptor signaling regulates cell growth and vulnerability to doxorubicin in bladder cancer. J Urol 188: 276-286, 2012.

21. Kashiwagi E, Ide H, Inoue S, Kawahara T, Zheng Y, Reis LO, Baras AS and Miyamoto H: Androgen receptor activity modulates responses to cisplatin treatment in bladder cancer. Oncotarget 7: 49169-49179, 2016.

22. Fabris L and Calin GA: Understanding the genomic ultraconservations: T-UCRs and cancer. Int Rev Cell Mol Biol 333: 159-172, 2017.

23. Olivieri M,Ferro M, Terreri S, Durso M, Romanelli A, Avitabile C, De Cobelli O, Messere A, Bruzzese D, Vannini I, et al: Long non-coding RNA containing ultraconserved genomic region 8 promotes bladder cancer tumorigenesis. Oncotarget 7: 20636-20654, 2016.

24. Li Y, Izumi K and Miyamoto $\mathrm{H}$ : The role of the androgen receptor in the development and progression of bladder cancer. Jpn J Clin Oncol 42: 569-577, 2012.

25. Shiota M, Kiyoshima K, Yokomizo A, Takeuchi A, Kashiwagi E Dejima T, Takahashi R, Inokuchi J, Tatsugami K and Eto M: Suppressed recurrent bladder cancer after androgen suppression with androgen deprivation therapy or 5alpha-reductase inhibitor. J Urol 197: 308-313, 2017.

26. Izumi K, Ito Y, Miyamoto H, Miyoshi Y, Ota J, Moriyama M, Murai T, Hayashi H, Inayama Y, Ohashi K, et al: Expression of androgen receptor in non-muscle-invasive bladder cancer predicts the preventive effect of androgen deprivation therapy on tumor recurrence. Oncotarget 7: 14153-14160, 2016.

27. Xiong Y, Wang L, Li Y, Chen M, He W and Qi L: The long non-coding RNA XIST interacted with MiR-124 to modulate bladder cancer growth, invasion and migration by targeting androgen receptor (AR). Cell Physiol Biochem 43: 405-418, 2017.

28. Calin GA, Liu CG, Ferracin M, Hyslop T, Spizzo R, Sevignani C, Fabbri M, Cimmino A, Lee EJ, Wojcik SE, et al: Ultraconserved regions encoding ncRNAs are altered in human leukemias and carcinomas. Cancer Cell 12: 215-229, 2007.

29. Chen L, Chang WC, Hung YC, Chang YY, Bao BY, Huang HC, Chung WM, Shyr CR and Ma WL: Androgen receptor increases CD133 expression and progenitor-like population that associate with cisplatin resistance in endometrial cancer cell line. Reprod Sci 21: 386-394, 2014 